\title{
Hierarchical Decomposition Thermodynamic Approach for the Study of Solar Absorption Refrigerator Performance
}

\author{
Emma Berrich Betouche ${ }^{1}$, Ali Fellah ${ }^{2}$, Ammar Ben Brahim ${ }^{2}$, Fethi Aloui ${ }^{3, *}$ and Michel Feidt ${ }^{4}$ \\ 1 GEPEA-UMR CNRS 6144 Laboratory, École des Mines de Nantes, \\ Energetic Systems and Environment Department, 4 Alfred Kastler Street BP 20722, \\ 44307 Nantes, France; emna.berrich@univ-nantes.fr \\ 2 Applied Thermodynamics Research Unit (99/UR/11-21), National Engineering School of Gabès, \\ Omar Ibn Elkhattab Street, 6029 Gabès, Tunisia; al.fellah@gmail.com (A.F.); \\ Ammar.Benbrahim@enig.rnu.tn (A.B.B.) \\ 3 LAMIH CNRS UMR 8201, Department of Mechanics, Valenciennes University, Campus Mont Houy, \\ F-59313 Valenciennes Cedex 9, France \\ 4 LEMTA, CNRS UMR 7563, ENSEM-INPL, Université de Lorraine, 2 Avenue de la Forêt of Haye BP 160, \\ 54504 Vandoeuvre-lès-Nancy, France; michel.feidt@univ-lorraine.fr \\ * Correspondence: Fethi.aloui@univ-valenciennes.fr; Tel.: +33-3-27-51-19-62; Fax: +33-3-27-51-19-61
}

Academic Editor: Kevin H. Knuth

Received: 29 October 2015; Accepted: 16 February 2016; Published: 4 March 2016

\begin{abstract}
A thermodynamic approach based on the hierarchical decomposition which is usually used in mechanical structure engineering is proposed. The methodology is applied to an absorption refrigeration cycle. Thus, a thermodynamic analysis of the performances on solar absorption refrigerators is presented. Under the hypothesis of an endoreversible model, the effects of the generator, the solar concentrator and the solar converter temperatures, on the coefficient of performance (COP), are presented and discussed. In fact, the coefficient of performance variations, according to the ratio of the heat transfer areas of the high temperature part (the thermal engine 2) $A_{h}$ and the heat transfer areas of the low temperature part (the thermal receptor) $A_{r}$ variations, are studied in this paper. For low values of the heat-transfer areas of the high temperature part and relatively important values of heat-transfer areas of the low temperature part as for example $A_{h}$ equal to $30 \%$ of $A_{r}$, the coefficient of performance is relatively important (approximately equal to $65 \%$ ). For an equal-area distribution corresponding to an area ratio $A_{h} / A_{r}$ of $50 \%$, the COP is approximately equal to $35 \%$. The originality of this deduction is that it allows a conceptual study of the solar absorption cycle.
\end{abstract}

Keywords: solar absorption refrigerators; endoreversible model; hierarchical decomposition; coefficient of performance

\section{Introduction}

The development of thermodynamic models for three sources systems for which one can hope better thermodynamic outputs is very interesting. The difference between real output and ideal output will be the lowest compared to that of the two source machines. The coefficient of performance (COP) of such commercial liquid absorption machines remain for the moment, according to the used process, of about 0.3 to 0.7. The reversible model, known as of Carnot model, presents an ideal case far from reality because it does not include the entropy effect. The coefficient of performance $\mathrm{COP}_{\text {Carnot }}$ relative to this model is higher than the real performance coefficients. Goth and Feidt [1] proposed optimal 
conditions for heat pump or a refrigeration cycle coupled with an endoreversible Carnot reverse machine. They determined the minimum electrical power to furnish from a thermal view point, for a maintained fixed useful heat flux for steady state conditions, and the optimal area allocation for finite machine dimension by considering that the total area of the machine is equal to the area of the heat compartment $\mathrm{A}_{\mathrm{h}}$ and the area of the cold one $\mathrm{A}_{\mathrm{r}}$ :

$$
A=A_{h}+A_{r}
$$

Wijeysandera [2] studied the internal irreversibility effect on three sources cycle performance, for a fixed solar radiation level value, using an irreversible model. He demonstrated that for a fixed cooling capacity, the COP has two values: The continuous section of the curves gives the physically meaningful COP values. The COP decreases because of the important heat quantity transferred from the high temperature source to the heat-sink reservoir. Kaushik et al. [3] studied the performance of irreversible cascaded refrigeration and heat pump cycles. They proposed a finite time thermodynamic model to optimize irreversible cascaded refrigeration/heat pump cycles constituted of finite heat capacitance thermal sources. They proposed an optimum COP expression. They demonstrated that external irreversibility is caused by heat sources and finite temperature differences, while, internal irreversibility is induced by the non-isentropic compression and expansion. The internal irreversibility effect is more significant than the external one.

\section{Thermodynamic Approach and Hierarchical Decomposition}

The solar absorption refrigeration cycle is presented on Figure 1. It is constituted by a thermal solar converter (TE1) and three main sources: a solar concentrator (hot source), an intermediate source, a cold source; as well as four essential elements which are: a generator, an absorber, a condenser and an evaporator.

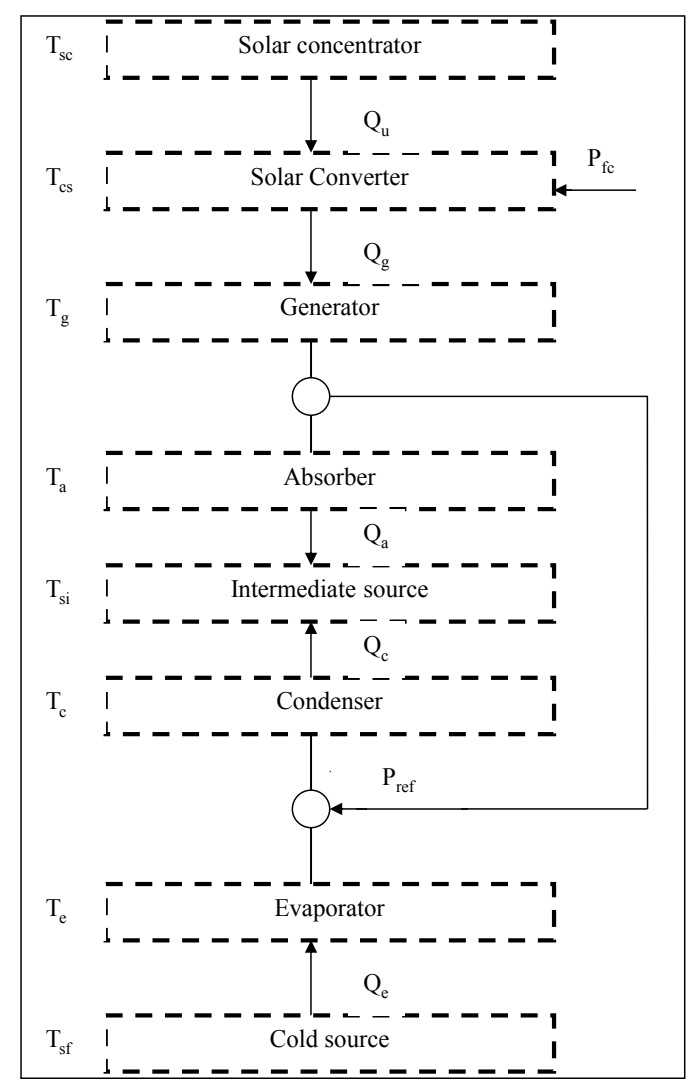

Figure 1. Equivalent model of the solar absorption refrigeration cycle. 
The general thermodynamic approach is based on the hierarchical decomposition which is usually used in mechanical structure engineering. Here, the methodology is applied to a solar absorption refrigeration cycle. The hypothesis of the endoreversible analyses are:

(1) The heat sources are thermostats (reservoirs with finite heat source capacitance).

(2) The heat transfers with the sources are supposed to be linear. This linear heat transfer law can be written as follows:

$$
Q=U A \Delta T
$$

where $U$ is the heat transfer coefficient, $A$ is the area and $\Delta T$ is the temperature difference according to a level or a sub-level.

Other linear heat transfer laws can be used. For example, Chen et al. [4] used the following linear law Equation (3) to optimize "four" heat source absorption refrigeration cycle using an endoreversible model:

$$
Q=U A \Delta T^{-1} \tau
$$

where $\tau$ is the cycle period. They thus proposed a relation between the COP and the cooling load. They found that when the "heat quantities of the condenser and the absorber ratio" increase, the COP increases for fixed "temperatures of the generator to the absorber ratio". There is an optimum refrigerator cooling load $Q_{e} / \tau$ corresponding to a COP approximately equals to $50 \%$, for any heat quantities of the condenser and the absorber ratio.

In our studied cases in this paper, the coefficient of performance will be presented and discussed for practical ranges of solar concentrator, generator, solar converter temperatures, at fixed entropy chosen for COP optimization. The COP evolution will be also illustrated function of the ratio of the heat transfer areas of the high temperature part $A_{h}$ and the heat transfer areas of the low temperature part (Thermal Receptor) $A_{r}$.

(3) Any inlet quantity to the system is supposed positive and any outlet quantity is negative (Convention).

(4) All the subsystems of the structure are endoreversible. Consequently, only the heat transfers between sub-systems, through the borders are sources of irreversibility.

The Lagrange multipliers method is used for the optimization. The optimization constraints are the thermodynamic laws. The Carnot model is an ideal model far from the reality as it doesn't take into account the entropy production. Although, the endoreversible model takes into account just the internal irreversibility of the cycle. Thus, the entropy production is minimized comparing to the irreversible model in which both the internal and external irreversibility are considered.

The hierarchical decomposition is usually used in mechanical structure engineering. Here, the methodology is applied to an absorption refrigeration cycle. A thermodynamic analysis of the influence of irreversibility on the refrigerators' performances is presented. The point of merit in this work is the application of hierarchical decomposition and the optimization by sub-structuring [5] and approaches which combine thermodynamic criteria and technical-economic criteria [6] for the study of the solar absorption cycle.

The equivalent model of a solar absorption refrigeration cycle is presented in Figure 1. The decomposition, is represented in Figure 2. It is constituted by four subdivision levels: The first level (L.I) is the compact global system (TE1 + TE2 + TR) which consists of two sublevels (N.II.1 and 2): the thermal converter (TE1) and the command and refrigeration system which is composed of two sublevels: the thermal engine TE2 and the thermal receptor TR. The last level (L.IV) regroups four main elements: generator, absorber, condenser and evaporator. The thermal engine TE2 is constituted by the generator and the absorber, while the thermal receptor TR consists of the condenser and the evaporator. 
Level I: Compact global System TE1+TE2+TR
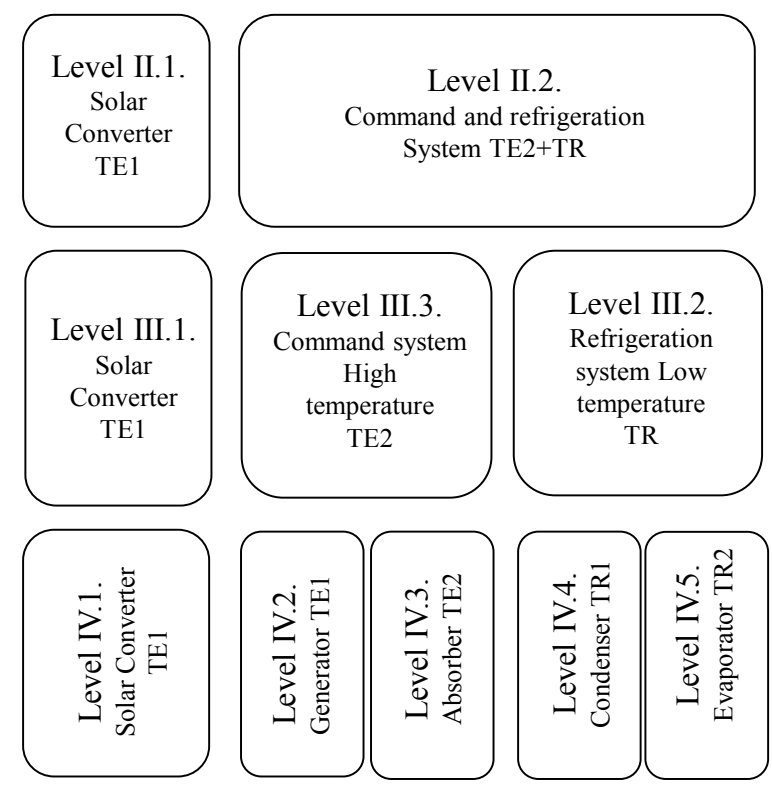

Figure 2. Hierarchical decomposition of the solar absorption refrigeration cycle.

Considering the reversibility inside the system, the first thermodynamic law [7-9] could be written as:

$$
Q_{e}+Q_{g}-Q_{a}-Q_{c}=0
$$

And the second thermodynamic law as:

$$
\frac{Q_{e}}{T_{e}}+\frac{Q_{g}}{T_{g}}-\frac{Q_{a}}{T_{a}}-\frac{Q_{c}}{T_{c}}=0
$$

The entropy production is written:

$$
S=\frac{Q_{a}+Q_{c}}{T_{s i}}-\frac{Q_{e}}{T_{e}}-\frac{Q_{g}}{T_{g}}
$$

The Lagrange multiplier method is the most employed method especially for the great systems, but this method requires an objective function and constraints.

According to the functional and conceptual unknowns' apparition in the mathematical model, the study could concern a sublevel, a level or a set of sublevels [10]. Mathematical equations present then some couplings between the optimal performance characteristics of the cycle. Let's consider, as an example, the subsystem formed by the refrigeration and control device (TE2 + TR). It is presented on Figure 3.

The Optimization Lagrange function presented in this section is specific to the case of "Level II.2" of the hierarchical decomposition: Refrigeration and control device (TE2+TR). The constraints are the first and the second thermodynamic laws. Thus, the Optimization Lagrange function can be written as follows [11]:

$$
L=Q_{j}+\lambda_{i}\left(\sum Q_{i}\right)+\lambda_{j}\left(\sum \frac{Q_{i}}{T_{i}}\right)
$$




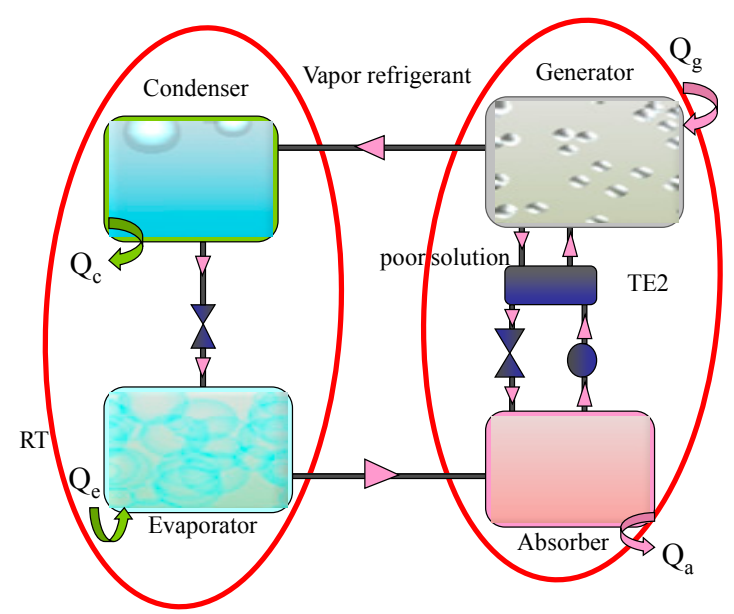

Figure 3. Refrigeration and control device.

The methodology has been applied to every level and sub-level of the whole decomposition. Significant relations are derived. They present the coupling of the optimal performance characteristics of the cycle. The effect of the external temperatures on the entropy production, performances and areas allocation is investigated.

\section{Results and Discussion}

\subsection{Solar Concentrator Effect on Entropy and Coefficient of Performance}

The entropy and the COP evolutions function of the solar concentrator $T_{s c}$ are illustrated on Figures 4 and 5 respectively, for fixed cold and intermediate source temperatures equal to $T_{s f}=10{ }^{\circ} \mathrm{C}$ and $T_{s i}=28{ }^{\circ} \mathrm{C}$, respectively. When the $T_{s c}$ increases of $45^{\circ} \mathrm{C}$, the entropy decreases approximately by $5 \%$ (Figure 3). As we can see on Figure 5, the COP has a real aspect. Its values varied from $28 \%$ to $58 \%$ which is a practical operating region. A $T_{s c}$ increase generates a net COP increase. A $T_{s c}$ variation of $35{ }^{\circ} \mathrm{C}$ (from $70{ }^{\circ} \mathrm{C}$ to approximately $112{ }^{\circ} \mathrm{C}$, for example) generates an increase of $20 \%$ on the COP. Indeed, the COP increases from $20 \%$ to approximately $42 \%$. Thus, we can conclude that the COP is very sensitive to the external sources temperatures. A 3\% decrease in the entropy can generate a COP increase of approximately $10 \%$. For high $T_{s c}$, the entropy production is minimized from $S=0.29 \mathrm{~kW} / \mathrm{K}$ to $S=0.25 \mathrm{~kW} / \mathrm{K}$, which generates a higher COP. Thus, a minimized entropy of $S \leqslant 0.25 \mathrm{~kW} / \mathrm{K}$, achieved for $T_{s c} \geqslant 105^{\circ} \mathrm{C}$ (Figure 4), corresponds to a COP $\geqslant 40 \%$ (Figure 5). In the following sections, the results are presented for $S=0.25 \mathrm{~kW} / \mathrm{K}$.

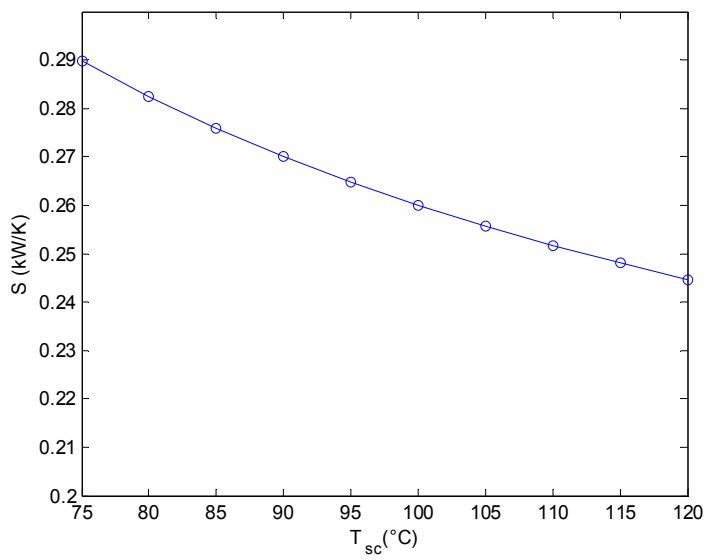

Figure 4. Solar concentrator temperature effect on the entropy $\left(T_{s i}=28^{\circ} \mathrm{C}, T_{s f}=10^{\circ} \mathrm{C}\right)$. 


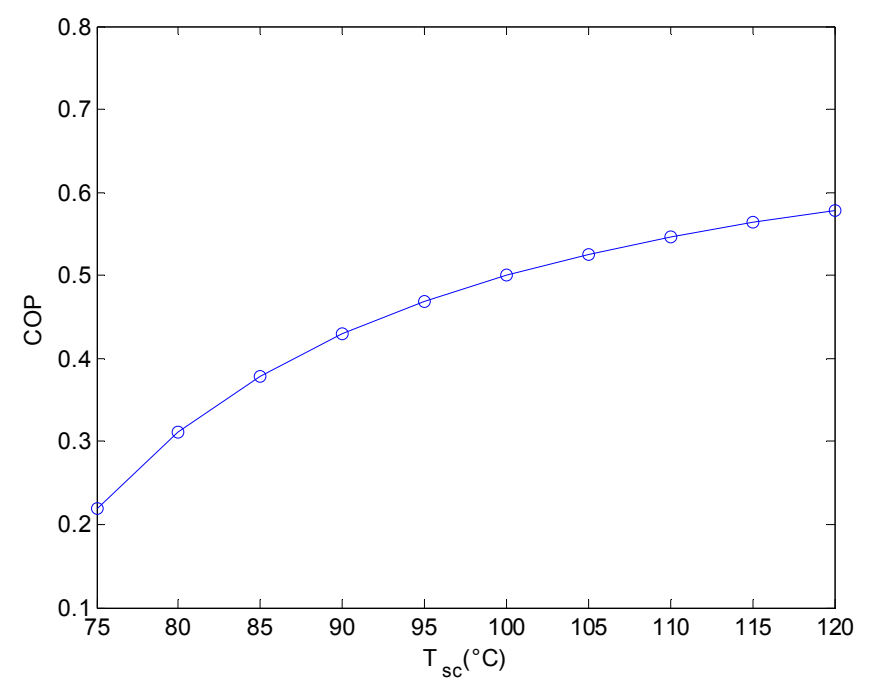

Figure 5. Solar concentrator temperature effect on the Coefficient of Performance for $\left(T_{s i}=28{ }^{\circ} \mathrm{C}\right.$, $\left.T_{s f}=10^{\circ} \mathrm{C}\right)$.

\subsection{Solar Converter Temperature Effect on the Coefficient of Performance}

Researchers usually look for the determination of machine efficiencies by considering different factor variations. The entropy production is strongly related to the cycle performance. The effects of the variation of the solar converter temperature, which is directly related to the heat source temperature $T_{c S}$, on the Coefficient of Performance COP for an entropy of $0.25 \mathrm{~kW} / \mathrm{K}$ is presented on Figure 6. We show that an increase in the solar converter temperature implies a clear increase in the COP. Indeed, for the work region presented, an increase of approximately $40{ }^{\circ} \mathrm{C}$ on the solar converter generates an increase of approximately about $28 \%$ of the COP. The different analysis discussed are in conformity with the work of Kaynakli et al. [12] who proposed a thermodynamic analysis of a water/lithium bromide absorption refrigeration cycle. In particular, they highlighted the effects of operating temperature and effectiveness of heat exchanger on the thermal loads of components, coefficient of performance and efficiency ratio. They concluded that the COP values increase with increasing generator and evaporator temperatures, but decrease while the condenser and absorber temperatures increase. They demonstrated that the efficiency ratio value varies with these temperatures. It should be noted that the generator temperature increases when the solar converter temperature increases.

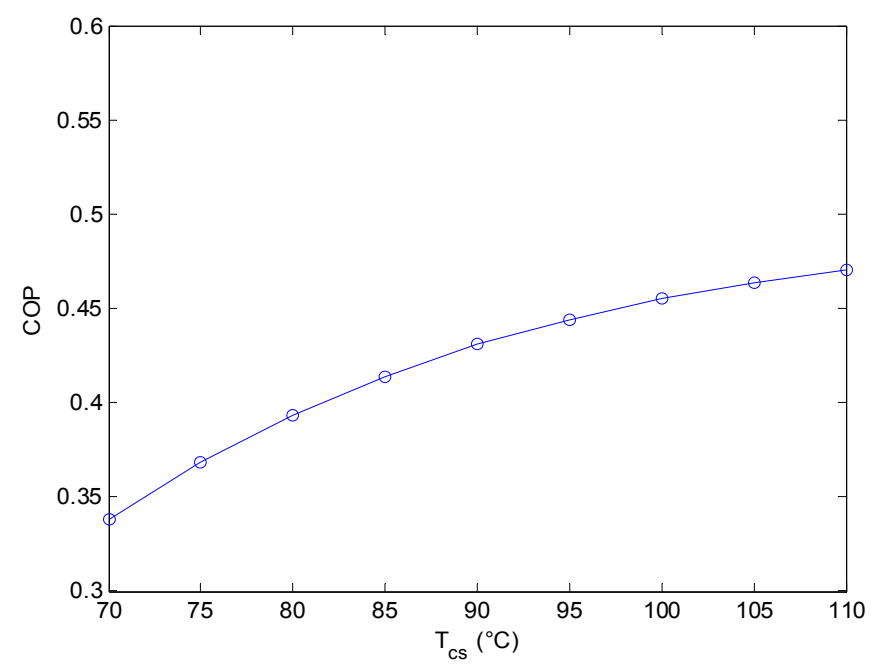

Figure 6. Effect of the solar converter temperature on the Coefficient of Performance. 


\subsection{Generator Temperature Effect on the Coefficient of Performance}

The generator temperature $T_{g}$ variation impact on the Coefficient of Performance COP for an entropy of $0.25 \mathrm{~kW} / \mathrm{K}$ is presented on Figure 7 . The COP increases from $48.7 \%$ to $51.7 \%$ when the generator temperature increases from $72{ }^{\circ} \mathrm{C}$ to $95^{\circ} \mathrm{C}$.

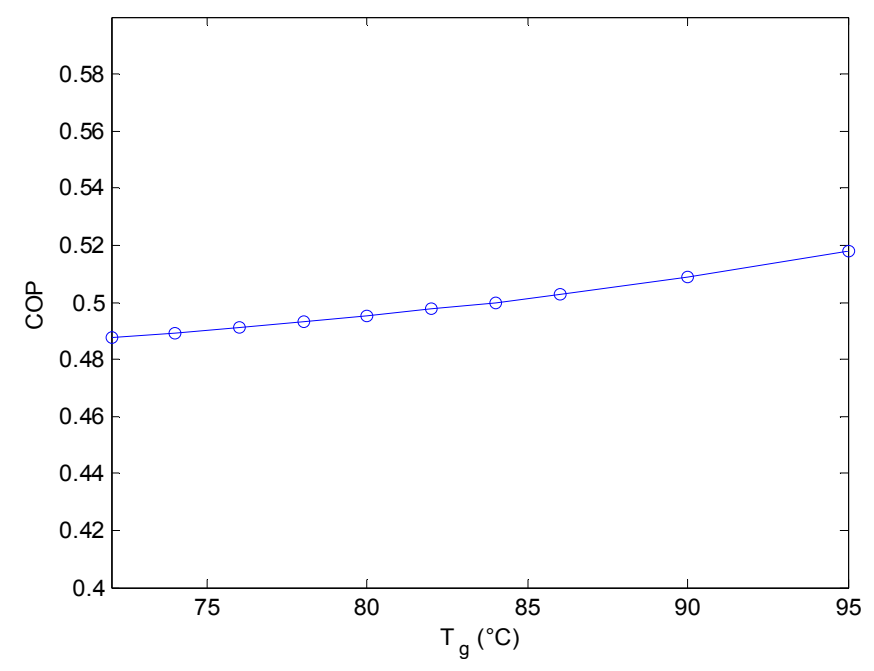

Figure 7. Effect of the generator temperature on the Coefficient of Performance.

These results are in conformity with the thermodynamic analysis of Kaynakli et al. [12] even if the parameters considered for both studies are slightly different. In fact, both of the studies illustrate the increasing evolution of the COP along the generator temperature range of $80^{\circ} \mathrm{C}$ to $95^{\circ} \mathrm{C}$. However, Kaynakli et al. [12] fixed the condenser and the evaporator temperatures to study the effect of the generator temperature on the COP, while we fixed the entropy production after an analysis of the evolution function of the solar concentrator for fixed cold and intermediate source temperatures at $10{ }^{\circ} \mathrm{C}$ and $28{ }^{\circ} \mathrm{C}$, respectively. Kaynakli et al. [12] found that, for $T_{g}$ between $80{ }^{\circ} \mathrm{C}$ and $95{ }^{\circ} \mathrm{C}$, the $\mathrm{COP}$ has an increasing evolution (as a function of the generator temperature) for a fixed condenser temperature and fixed evaporator temperature. The COP varied approximately between $60 \%$ and $71 \%$ for $T_{c}=40^{\circ} \mathrm{C}$ and $T_{e}=8{ }^{\circ} \mathrm{C}$; and between $78 \%$ and $80 \%$ for $T_{c}=35^{\circ} \mathrm{C}$ and $T_{e}=8{ }^{\circ} \mathrm{C}$ for $T_{g}$ between $80^{\circ} \mathrm{C}$ and $95^{\circ} \mathrm{C}$, which means less than a $2 \%$ of COP increase. It remains quasi-constant for higher values of $T_{g}\left(T_{g} \geqslant 95^{\circ} \mathrm{C}\right)$. In other words, a higher generator temperature doesn't necessarily provide higher COP, even if this contradicts the general rule that consists of increasing high command system temperatures to increase the COP. For our studied case, the generator temperature increase of $23^{\circ} \mathrm{C}$ generates a COP increase of approximately $3 \%$.

\subsection{Area Distribution Effect on the Coefficient of Performance}

The effect of the ratio of the heat transfer areas of the high temperature part $A_{h}$ and the heat transfer areas of the low temperature part (Thermal Receptor) $A_{r}$ on the COP is illustrated on Figure 8. For low values of the heat-transfer areas of the high temperature part and relatively important values of heat-transfer areas of the low temperature part as for example $A_{h}$ equal to $30 \%$ of $A_{r}$, the coefficient of performance is relatively important (approximately equal to $65 \%$ ). For an equal-area distribution corresponding to an area ratio $A_{h} / A_{r}$ of $50 \%$, the COP decreases approximately to $38 \%$. The originality of this deduction is that it allows a conceptual study of the solar absorption cycle. 


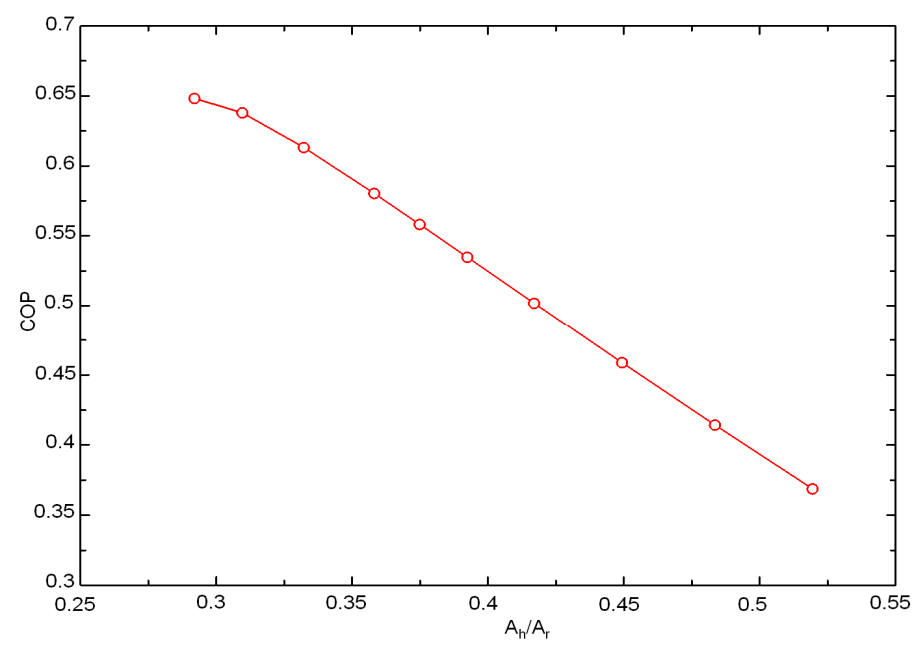

Figure 8. Effect of the areas distribution on the Coefficient of Performance.

\section{Conclusions}

A thermodynamic approach based on hierarchical decomposition was applied to an absorption refrigeration cycle. Under the hypothesis of the endoreversible analysis (heat sources are thermostats, heat transfers are supposed to be linear with sources, any inlet quantity to the system is supposed positive and any outlet one is negative (Convention), all the subsystems of the structure are endoreversible, and only the heat transfers between sub-systems, through the borders, are sources of irreversibility), functional and conceptual parameter effects on the COP were presented and discussed.

When the solar concentrator $T_{s c}$ increases to $45^{\circ} \mathrm{C}$, the entropy decreases by approximately $5 \%$. A $T_{s c}$ variation of $35^{\circ} \mathrm{C}$ generates an increasing of $20 \%$ on the COP. The COP has a real aspect. It varied from $28 \%$ to $58 \%$. A $3 \%$ decrease in the entropy can generate a COP increase of approximately $10 \%$. Minimized entropy of $S \leqslant 0.25 \mathrm{~kW} / \mathrm{K}$, attended for $T_{s c} \geqslant 105^{\circ} \mathrm{C}$, corresponds to a COP $\geqslant 40 \%$. An increase in the solar converter temperature implies a clear increase in the COP. Indeed, an increase of approximately $40{ }^{\circ} \mathrm{C}$ on the solar converter temperature generates an increase of about $28 \%$ of the COP with an entropy of $0.25 \mathrm{~kW} / \mathrm{K}$. A generator temperature increase of $23^{\circ} \mathrm{C}$ can generate an increase of $3 \%$ in the COP.

Acknowledgments: This work was supported by the National Engineering School of Gabés in TUNISIA.

Author Contributions: Emma Berrich Betouche realized this work, analyzed the results and wrote the paper. Ali Fellah contributed to the results analysis. He confirmed the approach on comparing it to his PhD thesis results. Ammar Ben Brahim supervised Berrich Betouche and Ali Fellah works. Michel Feidt and Fethi Aloui contributed to the results analysis and the paper revision.

Conflicts of Interest: The authors declare no conflict of interest.

\section{References}

1. Goth, Y.; Feidt, M. Conditions optimales de fonctionnement des pompes à chaleur ou machines à froid associeés à un cycle de Carnot endoreversible. CRAS 1986, 303, 19-24. (In French)

2. Wijeysundera, N.E. An irreversible thermodynamic model for solar powered absorption cooling systems. Sol. Energy 2000, 68, 69-75. [CrossRef]

3. Kaushik, S.C.; Kumar, P.; Jain, S. Performance evaluation of irreversible cascaded refrigeration and heat pump cycles. Energy Convers. Manag. 2002, 43, 2405-2424. [CrossRef]

4. Chen, L.; Zheng, T.; Sun, F.; Wu, C. Optimal cooling load and COP relationship of a four-heat-reservoir endoreversible absorption refrigeration Cycle. Entropy 2004, 6, 316-326. [CrossRef]

5. Meeuse, F.M. On the Design of Chemical Processes with Improved Controllability Characteristics; DUP Science: Delft, The Netherlands, 2002. 
6. Berrich, E.; Fellah, A.; Ben Brahim, A.; Feidt, M. Etude Conceptuelle et Fonctionnelle d'un Cycle de Réfrigération Par Absorption Solaire Selon l'Approche d'Endoréversibilité. In Proceedings of the 17th Annual SFT Conference on Efficacité Energétique, Vannes, France, 2009; pp. 695-700. (In French)

7. Fellah, A.; Ben Brahim, A.; Bourouis, M.; Coronas, A. Cooling loads analysis of an equivalent endoreversible model for a solar absorption refrigerator. Int. J. Exergy 2006, 3, 452-465. [CrossRef]

8. Zheng, T.; Chen, L.; Sun, F.; Wu, C. Performance optimization of an irreversible four-heat-reservoir absorption refrigerator. Appl. Energy 2003, 76, 391-414. [CrossRef]

9. Feidt, M. Thermodynamique et Optimisation Énergétique des Systèmes et Procédés; Lavoisier: Paris, France, 1987. (In French)

10. Berrich, E.; Fellah, A.; Ben Brahim, A.; Feidt, M. Conceptual and functional study of a solar absorption refrigeration cycle. Exergy 2011, 8, 265-280. [CrossRef]

11. Bejan, A. Method of entropy generation minimization, or modeling and optimization based on combined heat transfer and thermodynamics. Revue Générale de Thermique 1996, 35, 637-646. [CrossRef]

12. Kaynakli, O.; Kilic, M. Theoretical study on the effect of operating conditions on performance of absorption refrigeration system. Energy Convers. Manag. 2007, 48, 599-607. [CrossRef]

(C) 2016 by the authors; licensee MDPI, Basel, Switzerland. This article is an open access article distributed under the terms and conditions of the Creative Commons by Attribution (CC-BY) license (http:/ / creativecommons.org/licenses/by/4.0/). 